\section{Unmet Health Service Needs of Newly Diagnosed HIV-Pos- itive Men Who have Sex with Men in China: Results from An Ethnography}

\author{
Haochu (Howard) Li ${ }^{1 *}$, Joseph Lau ${ }^{2}$ and Eleanor Holroyd ${ }^{3}$ \\ ${ }^{1}$ School of Public Health, Shandong University, China \& UNC Project-Chi- \\ na, Institute of Global Health and Infectious Diseases, University of North \\ Carolina at Chapel Hill, USA \\ 2Jockey Club School of Public Health and Primary Care, The Chinese \\ University of Hong Kong, Hong Kong \\ ${ }^{3}$ School of Health Sciences, RMIT University, Australia
}

\begin{abstract}
We examined health services needs from the perspective of Newly diagnosed HIV-positive MSM (NHIVMSM) in Southern China. Forty one NHIVMSM took part in in-depth interviews. In addition, 20 health care providers and 16 volunteers were also interviewed in focus groups or individual face-to-face interviews. Participant observations in local healthcare settings, MSM venues and NGO offices were also conducted. Six thematic groupings of unmet health and social service needs were identified, such as information and guidance, improvement in quality of services, quality of and access to medication, enhancement of social support, social welfare and psychological and mental health. Implications for professional training in counseling, flexible free ART distribution, closer cooperation between formal and informal health care services, and establishment of community-based AIDS service organizations are discussed.
\end{abstract}

Keywords: China; Ethnography; Health care; HIV; MSM

\section{Introduction}

The trajectory of the HIV/AIDS has changed from a life-threatening illness/disease to a chronic condition [1]. Emerging needs among People Living With HIV (PLWH) need to be recognized as their utilization of primary care services has found to be associated with reduced risk of HIV transmission and improvement of disease outcomes [2]. Previous studies have investigated unmet needs for

*Corresponding author: Haochu (Howard) Li, School of Public Health, Shandong University, China \& UNC Project-China, Institute of Global Health and Infectious Diseases, University of North Carolina at Chapel Hill, USA, Tel: +86 1516913 2257; E-mail: hcli@alumni.cuhk.net

Citation: Li HH, Lau J, Holroyd E (2015) Unmet Health Service Needs of Newly Diagnosed HIV-Positive Men Who have Sex with Men in China: Results from An Ethnography. J AIDS Clin Res Sex Transm Dis 2: 004.

Received: July 08, 2015; Accepted: September 01, 2015; Published: September 15, 2015 clinical services among PLWH, such as viral load testing, CD4 count testing, or provision of Antiretroviral Therapy (ART) [3,4]. Understanding of holistic service needs for primary care such as risk counseling, case management, referrals and psycho-social care among PLWH [5] is warranted but remains under-studied.

In the international literature targeting PLWH, associations between socio-demographic factors and utilization of health-related services have been found. Mor and colleagues reported that those who are white, male, and non-intravenous drug users have higher rates of outpatient clinic/physician use, whereas those who are nonwhite, female, and intravenous drug users have higher rates of emergency room use [6]. Crook and colleagues found that those people who are single, lived along, and reported poorer health have a higher utilization of a community-based AIDS service organization [7]. Uphold and Mkanta conducted a literature review and found a list of factors related to use of health care services among PLWH, including predisposing factors (eg: race, gender and injection drug use), enabling factors (eg: insurance, social support systems and housing), clinical factors (eg: immune status symptoms and depression), and contextual factors (eg: characteristics of clinicians and urban/rural residence) [8]. Furthermore, unmet needs for family planning, sexually transmitted infection services, and combined and multiple services among PLWH were also described [9].

In China, it is estimated that there are 0.74 million PLWH in 2009 [10]. Service providers for PLWH are mainly clinicians who have not received training in providing psycho-social support [11] and are commonly overloaded in their working environment [12]. It has been contended that the high cost of care may further limit expansion of more integrated services [13]. One study estimated that the monetary cost of medical care was RMB 16,248 (USD2575) for asymptomatic PLWH and RMB 36,795 (USD5831) for AIDS patients using outpatient care and inpatient care services in China [14]. A study reported that the majority of the sampled PLWH desired group intervention as a means of psychological support [15]. Another study documented a limited utilization of HIV-related health services among HIV-positive MSM in Chengdu, China, such as $64.8 \%$ received medical treatment for their STD symptoms and 23.1\% referred their sexual partners for HIV antibody testing [16].

There is a paucity of data investigating unmet health service needs of newly diagnosed PLWH. The extent and quality of services provided to newly diagnosed PLWH is of particularly importance, as it affects subsequent mental health outcomes and HIV transmission risk [17-19]. Therefore, the aim of the current study is to explore health services needs from the perspective of newly diagnosed HIV-positive MSM in China.

\section{Methods}

\section{Settings}

In 2010 , Shenzhen has a population of 13.1 million, over $80 \%$ of which are internal migrants from other provinces or cities [20]. In 2010, about 100,000-200,000 MSM were estimated living in Shenzhen [21]. The first author conducted the fieldwork in Shenzhen from 
January to September 2010, in collaboration with a local Non-Government Organization (NGO) which had about 50 active members. The NGO worked closely with the Shenzhen Centre for Disease prevention and Control (CDC) to provide HIV/AIDS prevention services to MSM and was well connected to some groups of the PLWH.

\section{Participants}

We used purposive sampling [22] to recruit participants of diverse background characteristics (Table 1). Participants were at least 18 years old and had been diagnosed as HIV-positive in the last six months. A total of 31 participants were interviewed twice and 10 other men were interviewed once. All participants were internal migrants. In addition, we also recruited a convenience sample of twenty healthcare providers and sixteen associated volunteers. They were 6 staff members of the Shenzhen CDC, 4 staff members from the district CDC, and 4 staff members from Fang Bao Suo (a special grassroots branch in the Shenzhen CDC system) who were working on HIV/AIDS issues. Eleven of the staff members had face-to-face in-depth interviews, and three were in a group discussion. Six physicians in hospitals were in face-to-face in-depth interviews. For volunteers who had been involved in caring for HIV positive MSM, six of them were in a focus group discussion, and ten volunteers were in informal interviews.

\begin{tabular}{|c|c|c|c|}
\hline Characteristics & $\begin{array}{c}\text { Repeated interviews } \\
\qquad \mathrm{N}=31\end{array}$ & $\begin{array}{l}\text { One-time interview } \\
\qquad N=10\end{array}$ & Total $\mathbf{N}=\mathbf{4 1}(\%)$ \\
\hline \multicolumn{4}{|l|}{ Age } \\
\hline $18-25$ & 8 & 9 & $17(41.4)$ \\
\hline $26-35$ & 19 & 1 & $20(48.8)$ \\
\hline $36-40$ & 4 & 0 & $4(9.8)$ \\
\hline \multicolumn{4}{|l|}{ Duration since diagnosis } \\
\hline $1-2$ months & 15 & 3 & $18(43.9)$ \\
\hline 3-4 months & 11 & 4 & $15(36.6)$ \\
\hline $5-6$ months & 5 & 3 & $8(19.5)$ \\
\hline \multicolumn{4}{|l|}{ Occupations } \\
\hline Office & 10 & 1 & $11(26.8)$ \\
\hline Service/seller & 5 & 2 & $7(17.1)$ \\
\hline Laborer & 4 & 1 & $5(12.2)$ \\
\hline Sex worker & 1 & 6 & $7(17.1)$ \\
\hline Jobless & 2 & 0 & $2(4.9)$ \\
\hline \multicolumn{4}{|l|}{ Education } \\
\hline College & 8 & 3 & $11(26.8)$ \\
\hline High/technological school & 17 & 2 & $19(46.3)$ \\
\hline Secondary school & 6 & 4 & $10(24.4)$ \\
\hline Primary school or less & 0 & 1 & $1(2.5)$ \\
\hline \multicolumn{4}{|l|}{ Income (per month) } \\
\hline More than 6,000 & 3 & 0 & $3(7.3)$ \\
\hline $3,000-6,000$ & 13 & 5 & $18(43.9)$ \\
\hline Less than 3,000 & 14 & 2 & $16(39.0)$ \\
\hline \multicolumn{4}{|l|}{ Sexual identity } \\
\hline Homosexual & 27 & 5 & $32(78.1)$ \\
\hline Bisexual & 4 & 2 & $6(14.6)$ \\
\hline Heterosexual & 0 & 3 & $3(7.3)$ \\
\hline
\end{tabular}

Table 1: Summary of the characteristics of participants (NHIVMSM).

\section{Procedures}

When an MSM received confirmation about his HIV-positive status at the targeted CDC, our collaborator briefed them about the study and once consent had been obtained and a detailed briefing undertaken, referrals and appointments were then made. The interviews were conducted in a private room in this NGO and lasted 1.5 to two hours. Confidentiality, use of pseudonyms, safe storage of the data and the right to withdraw at any time were assured. Upon completion of the interview, a second interview was scheduled after three months. Participants were given a nominal reimbursement for participation in the form of cash. Ethical approval was granted by the Chinese University of Hong Kong. For health care providers and volunteers, one time interviews were conducted and each interview lasted one to 1.5 hours. In formal interviews, each participant received cash reimbursement for their participation. In informal interviews, no reimbursement was provided.

\section{Data analysis}

Tape-recorded interviews were transcribed verbatim in Chinese. Thematic content analysis was employed in the data analysis $[23,24]$. The analysis first involved preliminary reading of all the transcripts and field notes, and then identified recurring themes emerging from 
participants' reflections on unmet needs. Informative quotes relevant to the themes were grouped together. The themes were refined and merged or subdivided into groups, based on associations among the quotations. The thematic structures were further refined based on ethnographic observations. Data from HIV-positive MSM, volunteers and health care providers were triangulated. The first author translated the transcripts into English with selective sampling back translation employed, and the co-authors employed member checking of all emerging themes.

\section{Results}

\section{Needs for information and guidance}

In general, the sampled NHIVMSM were initially unaware of the availability of various HIV/AIDS-related health services and lacked experience of disease self management. There was hence a strong need for obtaining guidance from health professionals and fellow PLWH in order to better manage their life and illness progression.

Qi said, "I hope someone can provide us with advice because I am really unclear about this disease. A lot of stuff can be found on the internet, but such information is not necessarily professional. We need some professionals to provide us with the guidance, such as what matters need attention and how to help ourselves."

In a focus group involving six volunteers of the NGO, participants pointed out that HIV-positive MSM need to be provided with health-related information and knowledge. These volunteers mentioned: "We should provide more information about the benefits of health services to them, such as those of CD4 count testing" and "HIV-positive people need professional training (on how to manage their HIV/AIDS conditions)."

\section{Needs for improvement in quality of services}

Some respondents were dissatisfied with the quality of services provided by the CDC and the hospitals, and demanded improvement in these services, such as longer hours of service provision, protection of privacy and better attitudes among service providers. The attitudes of health care providers meant a lot to them. Some participants pointed out the need to improve the quality of the follow-up services.

Feng mentioned, "I had a CD4 count test this time and it was just 121. The staff in the office did not even remind me to take up ART. The CDC was irresponsible."

Sun said, "The services need to be improved...in terms of opening hours, as the clinic (AIDS clinic) only open in the mornings of Monday, Wednesday, Friday and Saturday. It's not convenient.”

Volunteers in a focus group discussion said, "Confidentiality is very important in the work related to PLWH...We must build up trust, and the first thing is confidentiality...we still need to learn and obtain training in this aspect."

\section{Needs for improvement in quality of and access to medication}

Nine participants were taking free ART and related free physical checks such as CD4 count and viral load testing. One participant (Kong) expressed a demand for free ART but was unable to obtain the free medicine. This is because Kong had neither a Shenzhen Hukou (registered permanent residence) nor a temporary residential permit and social insurance in Shenzhen, which were the eligible criteria for assessing free ART in Shenzhen CDC system. This policy therefore creates a barrier against access to treatment among internal migrant workers. Some participants demanded flexibility in accessing free ART.

Free ART was treated as an incentive to encourage HIV-positive MSM to contribute to their communities. One NGO leader stated, "We have a quota. ...If someone contributes to this population (MSM), we then provide him free ART. ...Because there is limited free ART, we need to encourage more PLWH...let them cherish the quota."

Participants testified that medicines of better quality were sought. For example, Jian said, "I don't know whether they are providing us with the most advanced medicines... What's the quality of these medicines? Every PLWH may want to know about this."

\section{Needs for enhancement of social support}

Social support was reported to be very important to the wellbeing of HIV-positive MSM, however these MSM interviewed testified to have very limited social support. Testimonies are as follows.

Xie said, "I have a lot of words from my heart. But I don't know whom I can talk to...I can only suppress myself, and I am very gloomy."

Feng said, "I hope to look for patients (HIV patients) in Shenzhen since they have experiences and know how to go through this. I don't know about where the (HIV-positive) groups are and therefore look for them on the Internet."

A senior volunteer said, "They (HIV-positive MSM) have no choice and life goes on (they need to survive in society). They isolate themselves. Sometimes when they disclose their seropositive status to someone, they become alienated gradually or broken off from their relationship."

\section{Needs for social welfare}

Many (20/41) participants did not have any kind of medical insurance. Many of them had to pay for all their health-related services. Some participants expressed their needs for social welfare, such as job referral, medical aid and higher basic living allowance.

Shen, a volunteer and a newly diagnosed HIV-positive MSM, described the importance in addressing the real hardship in the life of PLWH, "They (PLWH) are not satisfied with the society. We volunteers as a group can help each other and help people (PLWH) to look for a job, address their basic life needs, and change their living situation. These are realistic issues. Once they address these issues, they would not have this dissatisfaction."

Hei contracted a skin disease but he could not afford to see a doctor and he even did not have a basic medical insurance for migrant workers. Zhang stated that he would like to see that some fees for medical treatment waived, as a kind of medical fee assistance. The first author was told in a grassroots NGO that an international organization provided a quota based living allowance around RMB 2,000 (USD310) per person per month to PLWH in need. This allowance however only covered about a total of 20 PLWH, whereas there were currently almost six hundred PLWH (majority of them were MSM) in Shenzhen taking free ART.

\section{Psychological and mental health needs}

Thirty one participants experienced depressive symptoms, and twenty five participants experienced anxiety symptoms. Some 
participants stated an urgent unmet need for psychological and mental health services.

Xie said, "I am afraid that I cannot control myself. I always get lost in various fancies and conjectures. People like us need to receive psychological counseling. Migrant workers are usually in stressful situations...I am in the same situation, and what's worse, I also get HIV infection. I therefore feel very tired and very stressful... Once getting infected, migrant workers like us are generally passive. We need more psychological counseling, and more places we can look for help."

Shen said, "I feel PLWH really need a lot of care...I rely on myself adjusting (to the diagnosis). No one provided me a bit more help, and no one gave me special care. Actually I want so much to be cared, but there's nothing."

\section{Discussion}

The above identified needs reflect the complexity of health service concerns in the current HIV/AIDS-related health care system in China. In our fieldwork, the process of free Voluntary Counseling and Testing (VCT) was exhibited clearly in the CDC observed. Based on our fieldwork, however, this free VCT, a major service provided by CDC to the MSM population, was not well preformed. HIV testing played a much major role in service provision, while counseling was largely ignored. Practical problems of delivering VCT have also been presented in other studies, such as low utilization and access of VCT among MSM population [25] and the limited amount of pre and post test counseling conducted, poor accessibility and privacy protection, insufficient VCT staff, and irrelevant advocacy [26]. VCT should play an active role in providing professional and practical HIV/AIDS-related information and knowledge to enable NHIVMSM to manage their lives and illness progression. In order to address this unmet need, health care providers need more professional training in counseling, and structural collaborations with psychological and mental health institutes, as well as working closely with local NGOs in the provision of extended counseling services.

Other free services, such as free ART treatment and related physical check in AIDS clinic in D Hospital (an officially designated hospital for PLWH), and follow-up in CDC system, had limited service hours, concerns with respect to privacy and confidentiality, a paucity of follow-up, and reported indifference from the staff. The quality of free ART drugs was also an important concern, which is consistent with a previous study in China [27]. Side effects caused by these free ART drugs were reported quite often. Moreover, the Hukou-bounded free ART delivery was also problematic among migrants, since they generally drifted from one city to another and it was very inconvenient and costly for them to go back to their hometown to take free ART. Moreover, risk of being disclosed in their hometown was perceived as a serious issue, which prohibited them from applying for free ART. The effectiveness on HIV/AIDS preventive measures and intervention (eg: four free and one care) was therefore significantly hampered.

An implication is that health service delivery is influential in the effectiveness of these services. We would contend that the delivery of free ART can be reformed with the new national ID. This technical advance can facilitate a flexible free ART distribution. Migrant workers can use their ID to have free ART in their work place, and their information could be recorded and shared online within health care institutes. We would argue that transparency in the selection of free
ART drugs needs to be strengthened and a free-ART-drugs-updated scheme should be set up formally. Through this transparent scheme, the public (especially PLWH) is able to oversee how government and its organizations purchase ART drugs so as to insure affordable and quality ART drugs. The public, especially PLWH, Moreover, more systematic training of current CDC staff on how to provide high quality and effective health care to PLWH is needed. Implementation of free HIV/AIDS care should be linked to efforts to strengthen healthcare systems, and ensure long-term sustainability of funding and monitor equity of access to care [28].

Lack of psychological and mental health services was another repeatedly identified concern. Even though most participants experienced certain degree of psychological and mental health problems after being newly diagnosed as HIV-positive, both health care providers and these NHIVMSM presented less awareness of this issue. An implication is that the current bio medically -focused HIV/AIDS prevention and intervention measures need to be modified and psycho-social care and support aspects need to be strengthened. Chen and colleagues [29] have argued to set up better referral systems to facilitate inter-organizational communication and coordination.

Social and psychological support from peers and trustees were not well addressed, which implicates that networking between NHIVMSM and PLWH groups remains relatively weak. Mutual help and support groups and services were therefore relatively inaccessible. Moreover, formal health care (eg: CDC system and related hospitals) and informal health care (eg: grassroots organizations, support groups and peer networking) did not engage in close collaboration. Hence we further recommend closer structural and functional cooperation between health care institutes with grassroots MSM and PLWH organizations or groups, so that when MSM are newly diagnosed HIV-positive, they could be referred to support groups or organizations immediately to insure these men receive appropriate and effective health care and social support.

We found that many of the MSM interviewed did not have any social or commercial health insurance and need supportive social services, such as job security, medical aid, and basic living allowance. A Canadian study reported a reduced sense of isolation, increased knowledge of their illness and how to live with it, and improved quality of life as a result of using services from community-based AIDS organizations [7]. Studies show that receipt of support or ancillary services such as case management, mental health treatment, substance abuse treatment, housing assistance and transportation are significantly associated with early entry into and retention in HIV care [30]. We would further recommend that not-for-profit community-based AIDS service organizations should be established to target multiple needs of PLWH who are less likely to use mainstream health promotion and/or support services. Furthermore it is of importance that these services are primarily client-driven and are provided in all types of venues [31]. In the current study, biomedical services played a major role in HIV/AIDS prevention and intervention and had recently been substantially strengthened; however supportive social services remain largely underdeveloped, and therefore prohibit the effectiveness of HIV/AIDS prevention and intervention.

Several limitations to the present study should be noted. First, the themes analyzed are limited by the extent to which individuals disclosed the issues that were relevant and important to them. However, there still may be additional unmet needs that were not 
mentioned by these participants whom we interviewed. Second, by the nature of qualitative research, the sample size was small and sampling was limited to social networks of participants and local MSM/PLWH groups mainly confined to the author's ethnographic field. The results, therefore, were not generalizable to the total population of HIV-positive MSM in China. Third, although an interview outline was provided, in order to be mindful of the cultural context of discussing sensitive information, the first author liberally explored probes and themes, and hence, not all participants received the exactly same series of questions worded in the exact way or in the exact same order. Hence, data inconsistency could be a potential problem.

\section{Conclusion}

Despite these limitations, this is the first ethnographic study to address the unmet needs of newly diagnosed HIV-positive MSM in China. We come up with six unmet needs, which are related to information and guidance, the quality of services, the quality and accessibility of medication, social support, social welfare, and psychological and mental health. We have provided contextual insights to help inform the development of culturally sensitive and socially effective strategies for HIV prevention and intervention among MSM populations, which include strengthening counseling in VCT, building up a free-ART-drugs-updated scheme and a flexible free ART distribution, enhancing a closer cooperation between formal and informal health care services, and integrating supportive social services.

\section{Acknowledgements}

This research was funded by the Center for Health Behaviors Research of the Jockey Club School of Public Health and Primary Care (JCSPHPC), The Chinese University of Hong Kong (CUHK), a postgraduate study grant in JCSPHPC, and the Global Scholarship Programme for Research Excellence - CNOOC Grants 2010-11 in CUHK. The authors wish to thank all participants who shared their life histories and experiences, Shenzhen 258 Rainbow Workgroup, and Shenzhen CDC who helped significantly in the fieldwork and access to the populations.

\section{References}

1. Cooper V, Buick D, Horne R, Lambert N, Gellaitry G, et al. (2002) Perceptions of HAART among gay men who declined a treatment offer: preliminary results from an interview-based study. AIDS Care 14: 319-328.

2. Crepaz N, Lyles CM, Wolitski RJ, Passin WF, Rama SM, et al. (2006) Do prevention interventions reduce HIV risk behaviours among people living with HIV? A meta-analytic review of controlled trials. AIDS 20: 143-157.

3. Ikard K, Janney J, Hsu LC, Isenberg DJ, Scalco MB, et al. (2005) Estimation of unmet need for HIV primary medical care: a framework and three case studies. AIDS Educ Prev 17: 26-38.

4. Perkins D, Meyerson BE, Klinkenberg D, Laffoon BT (2008) Assessing HIV care and unmet need: eight data bases and a bit of perseverance. AIDS Care 20: 318-326.

5. Conviser R, Pounds MB (2002) The role of ancillary services in client-centred systems of care. AIDS Care 14: 119-131.

6. Mor V, Fleishman JA, Dresser M, Piette J (1992) Variation in health service use among HIV-infected patients. Med Care 30: 17-29.

7. Crook J, Browne G, Roberts J, Gafni A (2005) Impact of support services provided by a community-based AIDS service organization on persons living with HIVIAIDS. J Assoc Nurses AIDS Care 16: 39-49.
8. Uphold CR, Mkanta WN (2005) Review: use of health care services among persons living with HIV infection: state of the science and future directions. AIDS Patient Care STDS 19: 473-485.

9. Brickley DB, Almers L, Kennedy CE, Spaulding AB, Mirjahangir J, et al. (2011) Sexual and reproductive health services for people living with HIV: a systematic review. AIDS Care 23: 303-314.

10. Ministry of Health of the People's Republic of China (2010) China 2010 UNGASS Country Progress Report (2008-2009), China.

11. Chen WT, Starks H, Shiu CS, Fredriksen-Goldsen K, Simoni J, et al. (2007). Chinese HIV-positive patients and their healthcare providers: contrasting confusion versus western notions of secrecy and support. ANS Adv Nurs Sci 30: 329-342.

12. Xianyu Y, Lambert VA (2006) Investigation of the relationships among workplace stressors, ways of coping, and the mental health of Chinese head nurses. Nurs Health Sci 8: 147-155.

13. Jiang Z, Wang D, Yang S, Duan M, Bu P, et al. (2011) Integrated response toward HIV: a health promotion case study from China. Health Promot Int 26: 196-211.

14. Yang HM, Li J, Wu ZY, Xu LZ, Wang KA (2003). [Study on the utilization of health services and costs of hospital-based medical care for 29 patients with HIVIAIDS in China]. Zhonghua Liu Xing Bing Xue Za Zhi 24: 393-396.

15. Yu XN, Lau JT, Mak WW, Cheng YM, Lv YH, et al. (2009) Risk and protective factors in association with mental health problems among people living with HIV who were former plasma/blood donors in rural China. AIDS Care 21: 645-654.

16. Mi GD, Zeng YL, Huang WL, Zhang LL, Li T, et al. (2010) [A survey on the utilization of AIDS/STD related health services in HIV carriers of MSM, Chengdu city]. Zhongguo yufang yixue zazhi 4: 328-331.

17. Bova C, Burwick TN, Quinones M (2008) Improving women's adjustment to HIV infection: results of the Positive Life Skills Workshop Project. J Assoc Nurses AIDS Care 19: 58-65.

18. Crepaz N, Passin WF, Herbst JH, Rama SM, Malow RM, et al. (2008) Meta-analysis of cognitive-behavioral interventions on HIV-positive persons' mental health and immune functioning. Health Psychol 27: 4-14.

19. Fisher JD, Smith L (2009) Secondary prevention of HIV infection: the current state of prevention for positives. Curr Opin HIV AIDS 4: 279-287.

20. Shenzhen City Government General Office (2011) The $12^{\text {th }}$ Five years Shenzhen City Population Development Plan, China.

21. Xie W, Chen L, Cai W, Liu G, Zhao J, et al. (2010) Estimation of the population size of men who have sex with men in Shenzhen in 2010. Chinese Journal of AIDS and STD 17: 414-415.

22. Tongco MDC (2007) Purposive Sampling as a Tool for Informant Selection. Ethnobotany Research \& Applications 5: 147-158.

23. Pope C, Ziebland S, Mays N (2000) Qualitative research in health care. Analysing qualitative data. BMJ 320: 114-116.

24. Green J, Thorogood N (2009) Qualitative methods for health research, $\left(^{\text {nd- }}\right.$ edn). SAGE, London, UK.

25. Xu J, Zhang HB, Zheng YJ, Wang J, Zhu JL, et al. (2007) Demand and use of VCT service among MSM. Chinese Journal of Public Health 23: 1040-1042.

26. Huang T, Fu JH, Qian, YS, Wang TZ, Liao JZ (2007) Analysis of HIVIAIDS related VCT service in Shandong. Chinese Journal of AIDS \& STD 13: $430,431,465$.

27. Chen WT, Shiu CS, Simoni J, Fredriksen-Goldsen K, Zhang F, et al. (2009) Attitudes toward antiretroviral therapy and complementary and alternative medicine in Chinese patients infected with HIV. J Assoc Nurses AIDS Care 20: 203-217. 
28. Souteyrand YP, Collard V, Moatti JP, Grubb I, Guerma T (2008) Free care at the point of service delivery: a key component for reaching universal access to HIVIAIDS treatment in developing countries. AIDS 14: 161-168.

29. Chen WT, Shiu CS, Simoni J, Fredriksen-Goldsen K, Zhang F, et al. (2010) Optimizing HIV care by expanding the nursing role: patient and provider perspectives. J Adv Nurs 66: 260-268.
30. Conviser R, Pounds MB (2002) Background for the studies on ancillary services and primary care use. AIDS Care 1: 7-14.

31. Williams P, Narciso L, Browne G, Roberts J, Weir R, et al. (2005) Characteristics of people living with HIV who use community-based services in Ontario, Canada: implications for service providers. J Assoc Nurses AIDS Care 16: 50-63. 